\title{
Epimedium protects steroid-induced avascular necrosis of femoral head in rats by inhibiting autophagy
}

\author{
SU LIU $^{1}$, YUNZONG HUANG ${ }^{1}$, CHUANGLI WANG $^{1}$, SHOUJING TIAN $^{1}$, YOUJIA XU ${ }^{2}$ and JIANFEI GE ${ }^{1}$ \\ ${ }^{1}$ Department of Orthopedics, Zhangjiagang Hospital Affiliated to Soochow University, Zhangjiagang, Jiangsu 215600; \\ ${ }^{2}$ Department of Orthopedics, The Second Affiliated Hospital of Soochow University, Suzhou, Jiangsu 215004, P.R. China
}

Received April 9, 2018; Accepted September 24, 2018

DOI: $10.3892 /$ etm.2018.6827

\begin{abstract}
The effect of epimedium extracting solution on bone mineral density (BMD) of steroid-induced avascular necrosis of femoral head (SANFH) in rats was evaluated to further explore its function mechanism. Twenty-four Sprague-Dawley (SD) rats (male/female: 1/1) were randomly divided into three groups: the control $(n=8)$, the glucocorticoid $(n=8)$ and the epimedium $(n=8)$ group. Rats in the glucocorticoid and the epimedium group were injected with prednisolone acetate injection in gluteal muscles with $15 \mathrm{mg} / \mathrm{kg} /$ day twice a week. The epimedium group was given $10 \mathrm{ml} / \mathrm{kg}$ ephedra extracting solution containing crude drug with the concentration of $1.5 \mathrm{~g} / \mathrm{ml}$ daily by gavage. After 6 weeks, all the experimental rats were sacrificed and materials were extracted. The expression of autophagy-related proteins were detected by observing the bone of the femoral head. After comparison of the control group with the model group in BMD, it was found that there were significant differences $(\mathrm{P}<0.05)$. There were no significant differences between the control and the epimedium group $(\mathrm{P}>0.05)$. Neither between the glucocorticoid and the epimedium group $(\mathrm{P}<0.05)$. Epimedium extracting solution can significantly enhance the BMD of femoral heads, prevent osteoporosis and lead to collapse, increase the expression of apoptotic and protective proteins and reduce the expression of autophagy-related proteins, thus providing a preliminary theoretical study for the prevention and treatment of SANFH.
\end{abstract}

\section{Introduction}

Steroid-induced avascular necrosis of femoral head (SANFH) belongs to the non-traumatic type of avascular necrosis of femoral head. It refers to the pathological process where the high-dose glucocorticoids lead to death of active ingredients

Correspondence to: Dr Jianfei Ge, Department of Orthopedics, Zhangjiagang Hospital Affiliated to Soochow University, 68 Jiyang West Road, Zhangjiagang, Jiangsu 215600, P.R. China E-mail: bjenq356@163.com

Key words: steroid-induced avascular necrosis of femoral head, epimedium, autophagy, bone mineral density (osteocytes, bone marrow hematopoietic cells and adipocytes) of femoral heads (1). The main symptoms are regular pains in hip joints, joint dysfunction, limp and lower limb muscle atrophy, slow onset, long course of disease, high disability. This orthopedic disease is considered as one of the three major orthopedic problems (2). SANFH ranks first (2/3) of the non-traumatic avascular necroses, and the trend continues to increase (3), which has attracted great concerns of a large number of medical workers and scientists.

Modern medical science can generally be divided into the expectant and surgical treatment. Expectant treatments include painkillers, brake limbs, restricted load-bearing, and interventional therapy, but these methods do not acquire the desired effect. Surgical treatments are divided into the hip retention surgery and artificial joint replacement surgery (4). In recent years, based on the traditional Chinese medical theory and combined with the progress of modern medicine research, traditional Chinese medicine proposed the treatment based on syndrome differentiation from the overall treatment for alleviating pain and improving functions. This contributes to satisfactory results in the prevention and treatment of SANFH. Thus, we should pay attention to it (5).

Epimedium is a traditional Chinese medicine. Modern pharmacological experiments have shown that the main active ingredients of epimedium include total flavonoids of epimedium (TFE), and icariin (ICA). Epimedium participates in bone metabolism, stimulates bone cell proliferation and inhibits the activity of osteoclasts so as to prevent and cure osteoporosis $(6,7)$. In addition, it can also reduce the low, medium and high shear viscosity of the whole blood, improve blood rheology, and significantly reduce the platelet aggregation function (8), as well as regulate immune mechanism and improve immune system to prevent the primary disease.

Autophagy was initially discovered by a transmission electron microscopy. If cell autophagy occurs, vacuolar bilayer-like structures can be observed under the transmission electron microscopy. Sometimes autolysosomes and their residues can also be observed (9). Various cytokines and proteins such as Beclin1 and LC3 participate in cell autophagy to varying degrees (10). In recent years, with the deepening of research, many scholars have found and believed that glucocorticoid-induced bone autophagy is likely to play a role in the development of SANFH (11). Studies have shown that 
glucocorticoids cannot only induce osteoblast apoptosis, but also lead to the occurrence of bone autophagy (12). Excessive autophagy will cause harm to the body. Therefore, we believe that bone cell autophagy plays an important role in the pathogenesis of SANFH.

In this study, healthy Sprague-Dawley (SD) rats were used, and the early SANFH model was induced by intramuscular injection of prednisolone acetates. The mechanism of epimedium on the disease was observed from bone mineral density (BMD), immunohistochemical staining and other aspects, providing new drugs for clinical prevention and treatment of SANFH.

\section{Materials and methods}

Experimental animals and drugs. Twenty-four healthy SD rats weighing 200 $\pm 20 \mathrm{~g}$, provided by the Nanjing Qinglongshan Animal Experimental Center (Nanjing, China) of general standard feed. The rats were housed in a controlled room temperature $\left(21 \pm 2^{\circ} \mathrm{C}\right)$ on a $12: 12$-h light/dark cycle (lights on at 06:00). All rats had free access to food and water.

This study was approved by the Animal Ethics Committee of Soochow University Animal Center (Suzhou, China).

Acetic acid prednisolone injection was purchased from Zhejiang Xianju Pharmaceutical Co., Ltd. (Zhejiang, China); penicillin sodium injection was purchased from North China Pharmaceutical Co., Ltd. (Shijiazhuang, China); epimedium extracting solution was prepared by the liquid. A total of $1,080 \mathrm{~g}$ epimedium tablets were boiled and concentrated to $720 \mathrm{ml}$ solution (containing $1.5 \mathrm{~g} / \mathrm{ml}$ crude drugs). Primary mouse monoclonal B-cell lymphoma-2 (Bcl-2) antibody (dilution, 1:100; cat. no. sc-56015) and primary mouse monoclonal LC3 phosphatidylethanolamine conjugate (LC3II) antibody (dilution, 1:100; cat. no. sc-271625) were purchased from Santa Cruz Biotechnology, Inc. (Dallas, TX, USA). The remaining reagents were purchased from Nanjing Keygen Biotech Co., Ltd. (Nanjing, China).

Animal models and drug administration. According to the conversion method, rats in the glucocorticoid and epimedium groups were injected with $15 \mathrm{mg} / \mathrm{kg} /$ time prednisolone acetate injection in gluteal muscles twice a week. In order to prevent infection, $100,000 \mathrm{U} / \mathrm{kg} /$ time penicillin was injected intramuscularly after the injection of glucocorticoids twice a week. At the same time, the rats in the control group were given $10 \mathrm{ml} / \mathrm{kg}$ normal saline in gluteus muscles once a day for a total of 6 weeks.

At the same time, the epimedium group was given $10 \mathrm{ml} / \mathrm{kg}$ of epimedium extract with crude drug concentration of $1.5 \mathrm{~g} / \mathrm{ml}$, and the control and glucocorticoid groups received $10 \mathrm{ml} / \mathrm{kg}$ normal saline by gavage for 6 consecutive weeks. The rats received anesthesia with $10 \%$ chloral hydrate $(0.4 \mathrm{~g} / \mathrm{kg}$, i.p.) and then were sacrificed by cervical dislocation.

Hematoxylin and eosin $(H \& E)$ staining. The femoral head was put at one side, cut along the coronal plane and put into $10 \%$ formalin solution for the fixation for 1 week. Then the decalcification was performed by $5 \%$ nitric acid, and the femoral head was rinsed with water for $24 \mathrm{~h}$. After that, a series of alcohols were used for dehydration and the conventional paraffin embedding was performed. Afterwards, the head was sliced up and H\&E staining was conducted. Subchondral regions were regarded as film reading areas in each group. Pathological changes in bone trabeculae, bone marrow hematopoietic tissues, osteoblasts, osteocytes and adipocytes in the femoral bone and bone marrow tissues were observed under a light microscope (BX-42; Olympus, Tokyo, Japan).

Detection of BMD. After anesthesia by $10 \%$ chloral hydrate, the anatomy was conducted under sterile conditions, and the appearance, texture and articular cartilage colour were observed when taking the femoral head. We removed the surrounding muscles of the femoral head at one side and placed the specimen on the BMD instrument with a computer installed with bone density automatic measurement software to detect its BMD.

Immunohistochemical staining. We took the femoral head at one side, cut along the coronal plane and put it into $10 \%$ formalin solution for the fixation at $4^{\circ} \mathrm{C}$ for 1 week. Then the decalcification was performed by $5 \%$ nitric acid, and the femoral head was rinsed with water for $24 \mathrm{~h}$. After that, a series of alcohols were used for dehydration and the conventional paraffin embedding was performed. The head was sliced up. Then, after the dewaxing, hydration and antigen repairs, Bcl-2 or LC3-II antibodies were incubated at room temperature for $2 \mathrm{~h}$, washed with phosphate-buffered saline (PBS) 3 times and incubated for $10 \mathrm{~min}$ with biotin-labeled secondary antibodies. The head was rinsed again, re-stained it with hematoxylin stain and washed with running water for $1 \mathrm{~min}$. Subchondral regions were regarded as film reading areas in each group, and the staining intensity was observed under a light microscope (BX-42; Olympus).

Western blotting. Bone tissues (200 mg) were selected and weighed and then cut into pieces. These tissue pieces were placed into the pre-sterilization ceramic mortar, and the shredding tissues were quickly crushed by a grinding hammer with liquid nitrogen. After the liquid nitrogen fully volatilized, we added lysis solutions. The added lysis solutions were prepared as follows: radioimmunoprecipitation assay (RIPA) proteins containing phenylmethylsulfonyl fluoride (PMSF) were added into $100 \mathrm{mg}$ tissues. The supernatant was extracted after centrifugation at $10,000 \mathrm{x} \mathrm{g}$ at $4^{\circ} \mathrm{C}$ for $20 \mathrm{~min}$ to determine the protein concentration. After the installation of the electrophoresis plate, we produced gels, and added proteins in the upper sample wells. Then, we conducted the electrophoresis under constant pressure. After the electrophoresis, we placed polyvinylidene difluoride (PVDF) membranes covered by gels into the transfer buffer and transferred membranes at $0^{\circ} \mathrm{C}$ under constant pressure. After the transfer, the PVDF membrane was added with $5 \%$ skimmed milk powder and closed at room temperature for $1 \mathrm{~h}$. After that, the primary antibody (LC3II) was added and the PVDF membranes were incubated overnight at $4^{\circ} \mathrm{C}$. The next day, the membranes were washed with phosphate-buffered saline Tween-20 (PBST) 3 times, and then, the secondary rabbit anti-mouse (HRP) IgG antibody (dilution, 1:2,000; cat. no. ab6728; Abcam, Cambridge, MA, USA) was incubated at room temperature. One hour later, the membranes were washed again 3 times. Finally, we added the developing 
$\mathbf{A}$

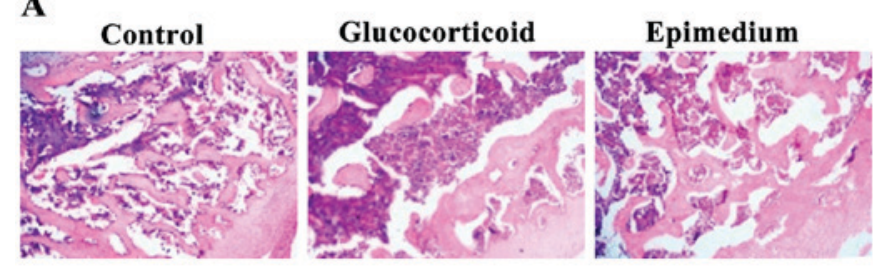

B

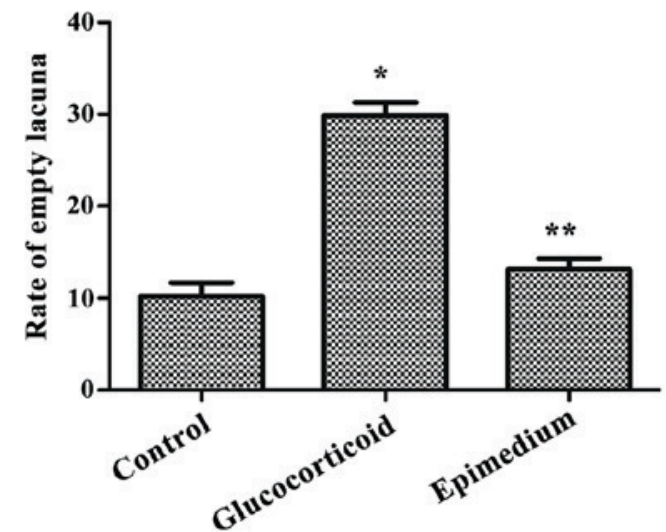

Figure 1. Observation of femoral head structures by H\&E staining. (A) Femoral head structures in the control, glucocorticoid and epimedium group are observed by H\&E staining images (x400). (B) According to the statistical data of the rate of empty lacunae, ${ }^{*} \mathrm{P}<0.05$, compared with the control group, while ${ }^{* *} \mathrm{P}<0.05$, compared with the glucocorticoid group. $\mathrm{H} \& \mathrm{E}$, hematoxylin and eosin.

Table I. Rat BMD detection results at 6 weeks (means \pm SD).

\begin{tabular}{lcccc}
\hline Groups & N & BMD $\left(\mathrm{g} / \mathrm{cm}^{2}\right)$ & F & P-value \\
\hline Control group & 8 & $0.204 \pm 0.013$ & 14.07 & $<0.001$ \\
Glucocorticoid group & 8 & $0.170 \pm 0.012^{\mathrm{a}}$ & & \\
Epimedium group & 8 & $0.195 \pm 0.008^{\mathrm{b}}$ & & \\
\hline
\end{tabular}

${ }^{a} \mathrm{P}<0.05$, compared with the control group $\mathrm{A}$; ${ }^{\mathrm{b}} \mathrm{P}<0.05$, compared with the glucocorticoid group B. BMD, bone mineral density.

liquid for exposure. The developing liquid was visualised using ECL kit (Merck Millipore, Billerica, MA, USA). The gray scale scan was conducted for the developing bands, and data were analyzed. $\beta$-actin served as internal control. Image J software (version 1.38; National Institutes of Health, Bethesda, MA, USA) was used for quantitative analysis of the blots.

Statistical analysis. Data statistical results were analyzed using Statistical Product and Service Solutions (SPSS) 13.0 software (SPSS, Inc., Chicago, IL, USA). Comparison between multiple groups was done using one-way ANOVA test followed by post hoc test (Least Significant Difference). $\mathrm{P}<0.05$ indicated that the difference was considered statistically significant.

\section{Results}

Glucocorticoid causes damage to BMD reduced by epimedium. The BMDs in femoral tissues of rats were measured by a BMD instrument. The results showed that there was
$\mathbf{A}$
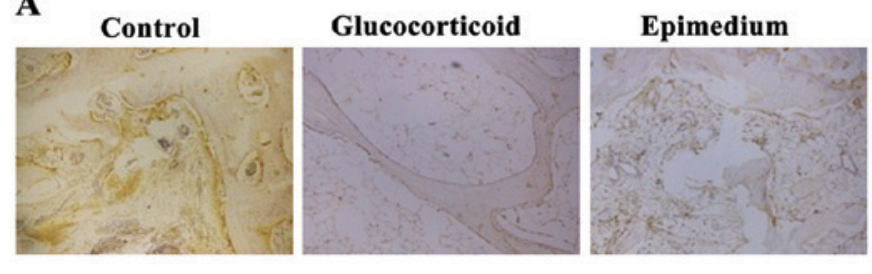

B

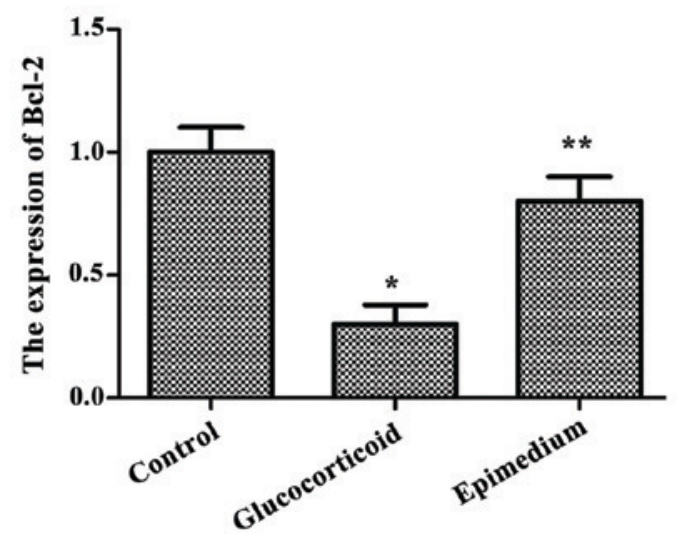

Figure 2. Observation of the expression of Bcl-2 of femoral heads by immunohistochemistry. (A) The expression of the apoptosis-related protective protein Bcl-2 of femoral heads is detected by immunohistochemical staining in the control, glucocorticoid and epimedium group. (B) The statistical data of the ratio of the color depth to that in the control group. ${ }^{*} \mathrm{P}<0.05$, compared with the control group, while ${ }^{* *} \mathrm{P}<0.05$, compared with the glucocorticoid group. Bcl-2, B-cell lymphoma-2.

significant difference between the control and glucocorticoid groups $(\mathrm{P}<0.05)$. There was no significant difference between the control and epimedium groups $(\mathrm{P}>0.05)$, while there was significant difference between the glucocorticoid and epimedium groups $(\mathrm{P}<0.05)$ (Table $\mathrm{I})$.

Glucocorticoid causes damage to bone tissues reduced by epimedium. Bone tissue structures were detected by H\&E staining. The results showed that in the control group, most trabecular bones were intact, thick, clear and regularly arranged with low content of adipocytes, while the content of hematopoietic cells was high. Some empty lacunae could be observed. In the glucocorticoid group the trabecular bones were thin and loose, and even the trabecular bone fractures were evident. Structures were irregular and unclear, and abundant hypertrophic adipocytes were observed in the medullary space. The content of hematopoietic cells was decreased, and empty lacunae occupied the full vision. The results of the epimedium group were very similar to those in the control group (Fig. 1A). After comparison between the control and glucocorticoid groups in empty lacuna rate, the results showed that the empty lacuna rate of the glucocorticoid group was $29.87 \pm 1.43 \%$, which was significantly higher than that in the control group $(10.23 \pm 1.45 \%)(\mathrm{P}<0.05)$. The empty lacuna rate of the epimedium group was $13.11 \pm 1.22 \%$, which was significantly different from that of the glucocorticoid group $(\mathrm{P}<0.05)$ (Fig. 1B). Therefore, the above results pathologically confirmed the differences between SANFH and normal femoral heads.

Glucocorticoid-induced bone cell apoptosis is reduced by epimedium. Immunohistochemistry results of Bcl-2 showed 
A
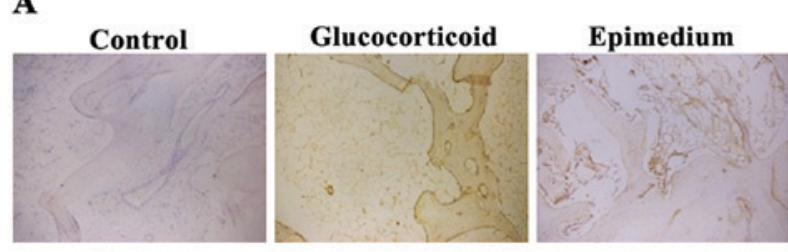

B

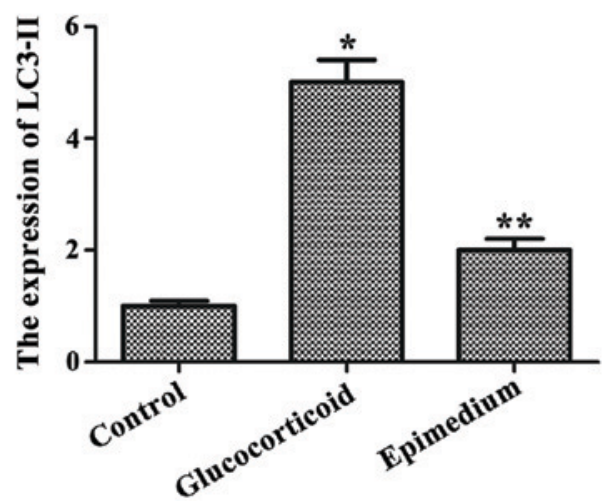

Figure 3. Observation of the expression of LC3-II by immunohistochemistry. (A) The expression of the autophagy-related protein LC3-II of femoral heads is detected by immunohistochemical staining in the control, glucocorticoid and epimedium group. (B) The statistical data of the ratio of the color depth to that in the control group. ${ }^{*} \mathrm{P}<0.05$, compared with the control group, while ${ }^{* *} \mathrm{P}<0.05$, compared with the glucocorticoid group.

that the most positive results were located in the tan or pale brown areas in the cytoplasm. The expression level of Bcl-2 in the glucocorticoid group was significantly lower than that in the control group, while the expression level of Bcl-2 in the epimedium group was significantly higher than that in the glucocorticoid group $(\mathrm{P}<0.05)$ (Fig. 2).

Glucocorticoid-induced autophagy-associated protein expression level is reduced by epimedium. Immunohistochemical results of LC3-II showed that the most positive results were located in the tan or pale brown areas in the cytoplasma, and there existed a small amount of positive particles in a small area of the nuclei. The expression level of LC3-II in the glucocorticoid group was significantly higher than that in the control group. The expression level of LC3-II was significantly lower in the epimedium group than that in the glucocorticoid group, and the differences were statistically significant $(\mathrm{P}<0.05)$ (Fig. 3).

Western blotting results showed that the concentration of autophagy-related protein Beclin1 and the ratios of LC3-II/LC3-I were significantly increased, and in the glucocorticoid group, the ratio of LC3-II/LC3-I was significantly lower than that in the epimedium group (Fig. 4A). The results of gray scale scan showed that the level of the autophagy-related protein was significantly higher in the glucocorticoid group than that in the control group, but the level of the autophagy-related protein in the epimedium group was significantly lower in the glucocorticoid group compared with the glucocorticoid group $(\mathrm{P}<0.05)$ (Fig. 4B).

\section{Discussion}

The first description of SANKF was recorded as early as the 1970s. Potter et al (13) first described the symptoms of femoral
A

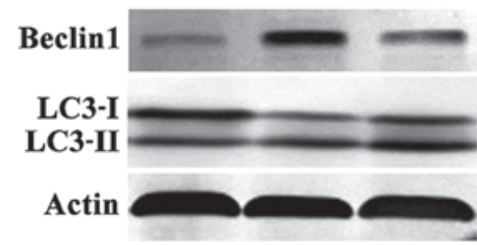

B
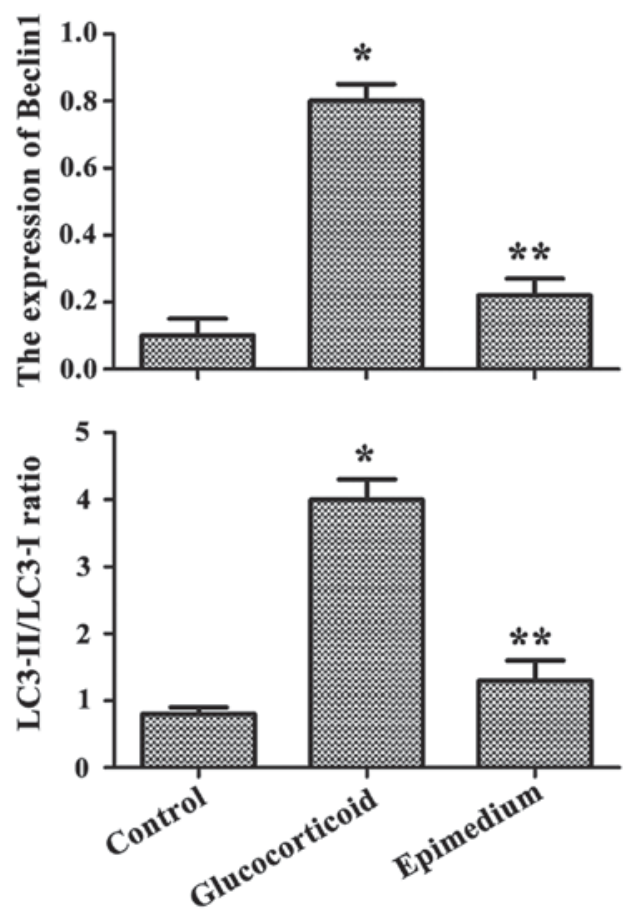

Figure 4. Detection of the expression of autophagy-related proteins by western blotting. (A) The expressions of the autophagy-related proteins Beclin1, LC3-I and LC3-II of femoral heads are detected by western blotting in the control, glucocorticoid and epimedium group. (B) The gray scale statistical data of Beclin1 proteins and the ratio of LC3-II/LC3-I gray scale. ${ }^{*} \mathrm{P}<0.05$, compared with the control group, while ${ }^{* *} \mathrm{P}<0.05$, compared with the glucocorticoid group.

head necrosis after extensive use of glucocorticoids in kidney transplant patients. Youm et al (14) studied 58 patients with different types of femoral head necrosis. The study found that patients with alcoholic avascular necrosis of femoral head and SANFH had high expression of apoptosis in the bone cells, but patients with idiopathic avascular necrosis of femoral head and traumatic avascular necrosis of femoral head have low expression of apoptosis. The present study supports the view that apoptosis plays an important role in the pathogenesis of steroid- and alcohol-induced avascular necroses of femoral heads. The H\&E staining results showed that the incidence rate of vacuolated lacunae in SANFH was significantly higher than that in the normal femoral head group. The results of immunohistochemistry showed that the expression level of Bcl-2 protein in SANFH was significantly lower than that in the control group. These data demonstrated that apoptosis was involved in the pathogenesis of SANFH.

Autophagic cell death is a newly discovered method of programmed cell death found in a molecular biology research. The process mainly relies on the degrading pathway of lysosomes to organelles or cytoplasmic components. It is a method by which cells decompose their own constituents so as to maintain a stable environment, and it is also a 
normal program of the body (9). Autophagy can be activated by various forms of cellular stresses, and when the cells are subjected to the above stresses, they initiate autophagy in order to survive (15). In the metabolic stress process, autophagy can produce adenosine triphosphates (ATPs) and macromolecules to provide energy resources for cells, thus enhancing cell viability, but when the cell stress is too strong or lasts for a long time, cells will increase the possibility of autophagic and programmed cell death (16). Autophagy not only plays an important role in the survival of cells, but also can promote cell death (17). Autophagy and apoptosis are not irrelevant to each other's life process; instead, the two are inextricably linked. Autophagy can cooperate with apoptosis to induce cells to enter the death program. Studies have shown that glucocorticoids can not only induce apoptosis in bone cells, but also can induce autophagy (18). These two processes are related to the dose of glucocorticoids. The activation of relatively low doses of glucocorticoids can promote autophagy, but that of relatively high doses of glucocorticoids enhance apoptosis. Autophagy is likely to act as a protective mechanism against cell death under stressful conditions with relatively short duration or less stresses. On the contrary, if stresses persist, autophagosomes accumulate and cause excessive autophagy, thus resulting in cell death. This study showed that autophagic apoptosis in SANFH exerted important effects on bone losses.

Pharmacological tests of modern Chinese medicine have confirmed that there are 74 active ingredients of epimedium, mainly including TFE, epimedium polysaccharide (EPS) and ICA (19). Modern pharmacological studies have shown that epimedium has a certain pharmacological effect on the blood system, immune system, cardiovascular and cerebrovascular system, including anti-inflammatory, anti-osteoporosis, and anti-aging (20). Epimedium has a unique advantage in the prevention and treatment of osteoporosis and it improves BMD (21). Studies have shown that epimedium can increase the density of rat femora at mRNA and protein level. It has been studied that different concentrations of epimedium glycosides promote the growth and proliferation of osteoblasts, and also enhance the osteoblastic activity of osteoblasts (7). In addition, it has been found that ICA can promote the expression of type I collagens in osteoblasts and the synthesis of osteocalcins, and stimulates osteoblast proliferation and differentiation cultured in vitro (22). This study found that epimedium could significantly alleviate SANFH and reduce autophagic apoptosis.

In conclusion, the epimedium extracting solution can significantly enhance BMD of the femoral heads, prevent the collapse caused by osteoporosis, increase the expression of apoptotic protective proteins and reduce the expression of autophagy-related proteins, thus providing a preliminary theoretical study for the prevention and treatment of SANFH.

\section{Acknowledgements}

Not applicable.

\section{Funding}

No funding was received.

\section{Availability of data and materials}

All data generated or analyzed during this study are included in this published article.

\section{Authors' contributions}

SL and JG designed the study and performed the experiments. SL, YH, ST and CW established the animal models. ST and YX collected the data. YH and CW analyzed the data. SL and JG prepared the manuscript. All authors read and approved the final manuscript.

\section{Ethics approval and consent to participate}

This study was approved by the Animal Ethics Committee of Soochow University Animal Center (Suzhou, China).

\section{Patient consent for publication}

Not applicable.

\section{Competing interests}

The authors declare no competing interests.

\section{References}

1. Drescher W, Schlieper G, Floege J and Eitner F: Steroid-related osteonecrosis - an update. Nephrol Dial Transplant 26: 2728-2731, 2011.

2. Indira D, Snehal S, Sudha CR and Raja Babu KK: Glucocorticosteroid-induced osteonecrosis: Lessons for the dermatologist (CME). Indian J Dermatol Venereol Leprol 66: 173-181, 2000.

3. Nowak DA and Yeung J: Steroid-Induced osteonecrosis in dermatology: A review. J Cutan Med Surg 19: 358-360, 2015

4. Tian ZJ, Liu BY, Zhang YT, Chen XZ, Qiao GY, Wang S and Ma ZL: MiR-145 silencing promotes steroid-induced avascular necrosis of the femoral head repair via upregulating VEGF. Eur Rev Med Pharmacol Sci 21: 3763-3769, 2017.

5. Tan XY, Gao FF, Gao ST, Liu YW, Chen XT and Liu LY: Treatment of steroid-induced osteonecrosis of femoral head by porous tantalum rod and gugutou huaisiyu capsule. Zhongguo Zhong Xi Yi Jie He Za Zhi 36: 40-43, 2016 (In Chinese).

6. Xu F, Ding Y, Guo Y, Liu B, Kou Z, Xiao W and Zhu J: Anti-osteoporosis effect of Epimedium via an estrogen-like mechanism based on a system-level approach. J Ethnopharmacol 177: 148-160, 2016.

7. Zhang D, Zhang J, Fong C, Yao X and Yang M: Herba epimedii flavonoids suppress osteoclastic differentiation and bone resorption by inducing $\mathrm{G} 2 / \mathrm{M}$ arrest and apoptosis. Biochimie 94: 2514-2522, 2012

8. Zhang WP, Bai XJ, Zheng XP, Xie XL and Yuan ZY: Icariin attenuates the enhanced prothrombotic state in atherosclerotic rabbits independently of its lipid-lowering effects. Planta Med 79: 731-736, 2013.

9. Shen HM and Mizushima N: At the end of the autophagic road: An emerging understanding of lysosomal functions in autophagy. Trends Biochem Sci 39: 61-71, 2014.

10. Wang Y, Liu J, Tao Z, Wu P, Cheng W, Du Y, Zhou N, Ge Y and Yang Z: Exogenous HGF prevents cardiomyocytes from apoptosis after hypoxia via up-regulating cell autophagy. Cell Physiol Biochem 38: 2401-2413, 2016.

11. Eberhardt AW, Yeager-Jones A and Blair HC: Regional trabecular bone matrix degeneration and osteocyte death in femora of glucocorticoid-treated rabbits. Endocrinology 142: 1333-1340, 2001.

12. Xia X, Kar R, Gluhak-Heinrich J, Yao W, Lane NE, Bonewald LF, Biswas SK, Lo WK and Jiang JX: Glucocorticoid-induced autophagy in osteocytes. J Bone Miner Res 25: 2479-2488, 2010. 
13. Potter DE, Genant HK and Salvatierra O: Avascular necrosis of bone after renal transplantation. Am J Dis Child 132: 1125-1129, 1978.

14. Youm YS, Lee SY and Lee SH: Apoptosis in the osteonecrosis of the femoral head. Clin Orthop Surg 2: 250-255, 2010.

15. Boya $\mathrm{P}$, Reggiori $\mathrm{F}$ and Codogno P: Emerging regulation and functions of autophagy. Nat Cell Biol 15: 713-720, 2013

16. Kepp O, Galluzzi L, Lipinski M, Yuan J and Kroemer G: Cell death assays for drug discovery. Nat Rev Drug Discov 10: 221-237, 2011.

17. Gurusamy N and Das DK: Is autophagy a double-edged sword for the heart? Acta Physiol Hung 96: 267-276, 2009.

18. Jia J, Yao W, Guan M, Dai W, Shahnazari M, Kar R, Bonewald L, Jiang JX and Lane NE: Glucocorticoid dose determines osteocyte cell fate. FASEB J 25: 3366-3376, 2011.

19. Zeng S, Xiao G, Guo J, Fei Z, Xu Y, Roe BA and Wang Y: Development of a EST dataset and characterization of EST-SSRs in a traditional Chinese medicinal plant, Epimedium sagittatum (Sieb. Et Zucc.) Maxim. BMC Genomics 11: 94, 2010.
20. Zhai YK, Guo X, Pan YL, Niu YB, Li CR, Wu XL and Mel QB: A systematic review of the efficacy and pharmacological profile of Herba Epimedii in osteoporosis therapy. Pharmazie 68: 713-722, 2013

21. Zhang DW, Cheng Y, Wang NL, Zhang JC, Yang MS and Yao XS: Effects of total flavonoids and flavonol glycosides from Epimedium koreanum Nakai on the proliferation and differentiation of primary osteoblasts. Phytomedicine 15: 55-61, 2008.

22. Chen KM, Ma HP, Ge BF, Liu XY, Ma LP, Bai MH and Wang Y: Icariin enhances the osteogenic differentiation of bone marrow stromal cells but has no effects on the differentiation of newborn calvarial osteoblasts of rats. Pharmazie 62: 785-789, 2007.

This work is licensed under a Creative Commons

Attribution-NonCommercial-NoDerivatives 4.0 International (CC BY-NC-ND 4.0) License. 